\title{
Förderung der Gewichtsabnahme klappt auch bei psychisch Kranken
}

Fragestellung: Inwieweit hilft eine maßgeschneiderte Verhaltenstherapie, übergewichtigen Menschen mit einer schweren psychischen Erkrankung beim Abnehmen?

Hintergrund: Übergewicht und Adipositas sind bei Personen mit ernsthaften psychischen Erkrankungen epidemisch verbreitet. Trotzdem schließen Studien zur Gewichtsreduktion diese Personengruppe mit ernsthaften psychischen Erkrankungen systematisch aus. Interventionen des Lebensstils bedürfen bei dieser Personengruppe einer besonderen Anpassung, da bei ihnen häufig psychiatrische Symptome und eine verminderte Kognition vorliegen.

Methodik: Die Autoren rekrutierten übergewichtige und adipöse Erwachsene aus 10 kommunalen psychiatrischen ambulanten Rehabilitationsprogrammen. Sie wurden randomisiert einer Interventions- oder Kontrollgruppe zugeteilt. In der Interventionsgruppe wurden sowohl maßgeschneiderte Gruppenund individuelle Gewichtsmanagment-Sitzungen als auch Übungsprogramme in der Gruppe durchgeführt. Die Gewichtsveränderung wurde nach 6, 12 und 18 Monaten untersucht. Von den 291 randomisierten Teilnehmern hatten 58,1\% eine Schizophrenie oder eine schizoaffektive Störung, 22,0\% hatten eine bipolare Erkrankung und 12\% eine schwere Depression. $\mathrm{Zu}$ Beginn, war der mittlere BMI $\left(\mathrm{kg} / \mathrm{m}^{2}\right) 36,3$, das mittlere Gewicht lag bei 102,7 kg (225,9 Pfund). Von 279 Probanden wurde das Gewicht nach 18 Monaten bestimmt.

Ergebnisse: Der Gewichtsverlust in der Interventionsgruppe verstärkte sich über die 18-monatige Studiendauer und unter-

Daumit GL, Dickerson FB, Wang NY et al. A behavioral weightloss intervention in persons with serious mental illness. N Engl J Med 2013;368(17):1594-602. schied sich signifikant von der Kontrollgruppe bei jeder der Visiten. Zum Zeitpunkt 18 Monate betrug die mittlere Gewichtsdifferenz zwischen den beiden Gruppen

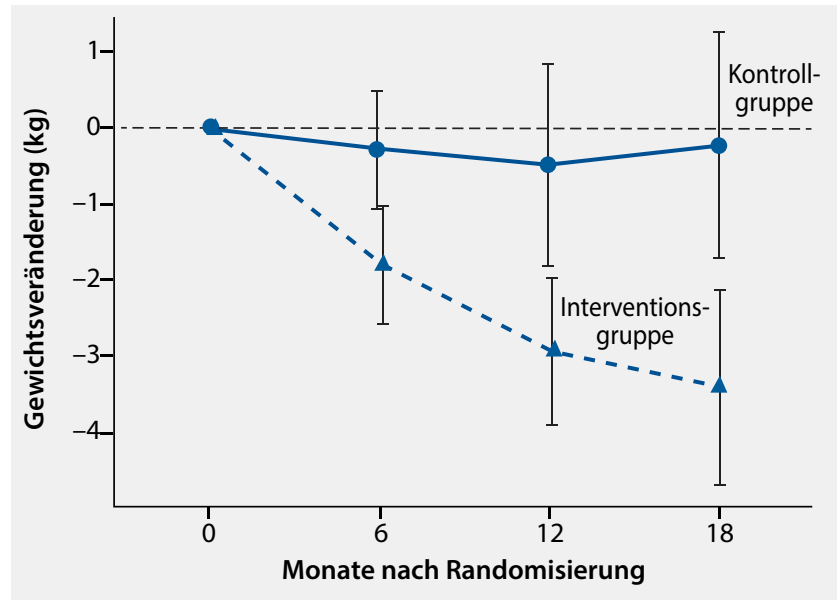

1 Mittlere Gewichtsveränderung bei der Interventions- und der Kontrollgruppe.

$-3,2 \mathrm{~kg}(-7,0 \mathrm{lb}, \mathrm{p}=0,002)$, $>$ Abb. 1; 37,8\% der Personen in der Interventionsgruppe reduzierten ihr Gewicht um mindestens 5\% des Ausgangsgewichts, während dies nur bei 22,7\% der Kontrollgruppe erreicht wurde $(\mathrm{p}=0,009)$. Es gab keine signifikanten Unterschiede im Auftreten von unerwünschten Ereignissen.

Schlussfolgerungen: Eine interventionelle Verhaltenstherapie führte zu einer signifikant besseren Gewichtsreduktion bei Personen mit ernsthaften psychiatrischen Erkrankungen und Übergewicht oder Adipositas. Wenn man die Häufigkeit des hohen Vorkommens von Übergewicht und Adipositas in dieser Personengruppe betrachtet, bedeuten die hier erhobenen Ergebnisse, dass die interventionelle Verhaltenstherapie zur Gewichtsreduktion bei Personen mit ernsthaften psychischen Erkrankungen bei dieser Hochrisikogruppe implementiert werden sollte.

\section{- Kommentar von Prof. Dr. med. Nanette C. Schloot}

\section{Kontinuierlich anhaltender, statt zu Beginn verstärkter Gewichtsverlust}

Diese Arbeit zeigt, dass auch bei psychiatrisch Erkrankten die verhaltensbasierte Lebensstilintervention zur Gewichtsreduktion eingesetzt werden kann und bei einem hohem Prozentsatz von knapp 40\% das Gewicht um mindestens 5\% reduziert. Die gewählte Intervention bei den Patienten mit einem mittleren BMI von $36 \mathrm{~kg} / \mathrm{m}^{2}$ entsprach im Wesentlichen den Empfehlungen für nicht psychiatrisch Erkrankte und schloss sowohl eine Ernährungsumstellung als auch eine gesteigerte körperliche Ak- tivität in Einzel- und Gruppensitzungen mit ein. Die Kontrollgruppe erhielt Ernährungsempfehlungen und die Aufforderung, sich vermehrt körperlich zu betätigen. Im Gegensatz zu den Erfahrungen mit psychisch gesunden adipösen Menschen, bei denen häufig initial ein besonders starke Gewichtsreduktion erreicht wird, dessen Verlauf dann abflacht, kam es bei der aktuellen Studie zu einer kontinuierlich anhaltenden Gewichtsreduktion. 